\title{
Fetal Heart Tone Location
}

National Cancer Institute

\section{Source}

National Cancer Institute. Fetal Heart Tone Location. NCI Thesaurus. Code C92792.

The site of fetal cardiac sounds that can be distinctly detected in relation to the maternal abdomen. 